\title{
Estudio Fitoquímico y Evaluación del Potencial Leishmanicida de la Especie Esenbeckia litoralis (Rutaceae)
}

\author{
Fernis J. Marin ${ }^{(1,2)}$, Omar L. Torres ${ }^{(1,2) \star}$, Gilmar G. Santafé(2) y Sara M. Robledo(3) \\ (1) Fac. de Ciencias de la Salud, Dpto. Regencia y Farmacia, Univ. de Córdoba, Cra 6 No. 76-103, \\ Montería-Colombia. (e-mail: fernismarin@hotmail.com; omart2365@gmail.com) \\ (2) Fac. de Ciencias Básicas, Dpto. de Química, Univ. de Córdoba, Cra 6 No. 76-103, Montería-Colombia. \\ (e-mail: gsantafe@correo.unicordoba.edu.co) \\ (3) PECET-Instituto de Investigaciones Médicas, Fac. de Medicina Univ. de Antioquia, Calle 62 No $52-59$ \\ SIU Laboratorio 632, Medellín-Colombia. (e-mail: sara.robledo@udea.edu.co)
}

* Autor a quien debe ser dirigida la correspondencia.

Recibido Jul. 10, 2015; Aceptado Ago. 28, 2015; Versión final Oct. 20, 2015, Publicado Feb. 2016

\begin{abstract}
Resumen
Se realizó el estudio fitoquímico y la evaluación de la actividad leishmanicida de la especie Esenbeckia litoralis (Rutaceae) para obtener metabolitos secundarios mayoritarios con potencial biológico. Los extractos obtenidos se fraccionaron empleando técnicas cromatográficas y los compuestos se identificaron mediante el análisis de datos espectroscópicos: Infrarrojo, Resonancia Magnética Nuclear y Espectrometría de Masas; la actividad leishmanicida se determinó por citometría de flujo y la citotoxicidad por el método MTT. Se aislaron e identificaron siete compuestos: Flindersiamina (1), 1-hidroxi-3-metoxi$N$-metilacridona (2), Maculosidina (3), Kokusaginina (4), Maculina (5), Dictamina (6) y Gardenina B (7). El compuestos 2 y el subextracto en acetato de etilo de corteza mostraron una moderada actividad frente a Leishmania (V) panamensis con un $\mathrm{IC}_{50}$ de 37.5 y $9.2 \mu \mathrm{g} / \mathrm{ml}$ respectivamente. El estudio muestra la presencia de metabolitos activos con potencial leishmanicida en estos extractos, lo que podría representar una alternativa terapéutica para el tratamiento de la enfermedad parasitaria conocida como leishmaniosis.
\end{abstract}

\section{Phytochemical Study and Evaluation of the Leishmanicidal Potential of the Species Esenbeckia litoralis (Rutaceae)}

\begin{abstract}
A phytochemical study and evaluation of leishmanicidal activity of Esenbeckia litoralis species (Rutaceae) to obtain secondary metabolites with high biological potential were carried out and completed. The obtained extracts were fractionated using chromatographic techniques and the compounds were identified by spectroscopic analysis including infrared, nuclear magnetic resonance and Mass Spectrometry; the leismanicidal activity was determined by flow cytometry and cytotoxicity by MTT method. Seven compounds were isolated and identified: Flindersiamine (1), 1-hydroxy-3-methoxy- $N$-methylacridone (2), Maculosidine (3), Kokusaginine (4), Maculine (5), Dictamine (6) and Gardenin B (7). The compound 2 and the ethyl acetate extract of bark showed moderate activity against the parasite of Leishmania panamensis with an $\mathrm{IC}_{50}=37.5$ and $9.2 \mathrm{ug} / \mathrm{ml}$ respectively. The study showed the presence of active metabolites with leishmanicidal potential in these extracts, which could represent an alternative therapy for treatment of the leishmaniasis.
\end{abstract}

Keywords: Rutaceae; Esenbeckia litoralis; phytochemistry; alkaloid; leishmanicidal activity 


\section{INTRODUCCIÓN}

La leishmaniasis constituye un grupo de enfermedades parasitarias, endémicas en 98 países, que afecta anualmente a 1,3 millones de personas y es la segunda infección parasitaria en el mundo con alrededor de 50.000 muertes anuales (Alvar et al., 2012). En Colombia, durante el año 2014 se registraron 10.195 casos de leishmaniasis cutánea siendo la forma clínica más prevalente en el país y para el año 2015 se han reportado 5848 nuevos casos en lo que va corrido del año (Instituto Nacional de Salud, 2015). Se estima que el $91 \%$ de los municipios en Colombia que se encuentran por debajo de 1750 m.s.n.m han presentado casos de leishmaniasis, notándose un incremento gradual en la transmisión de la enfermedad en las ciudades, por el desplazamiento de los vectores y de las poblaciones a áreas semirurales (Segura et al, 2013).

Cabe destacar que Leishmania (V) panamensis junto con $L$. (V) braziliensis son las especies de Leishmania más prevalentes en Colombia (Ovalle et al, 2006). A pesar de la utilización de tratamientos a base de antimoniales pentavalentes (Estibogluconato de sodio y el antimoniato de $\mathrm{N}$-metilglucamina), Mitelfosina y Anfotericina B (Pulido et al., 2012), los casos de leishmaniasis se han ido incrementando considerablemente, demostrando ineficacia de estos medicamentos, trayendo como consecuencia la modificación del tratamiento en aumentos de la dosis y conllevando a trastornos clínicos como efectos adversos. Toda esta problemática sumada a los fenómenos de resistencia del parásito al medicamento y a la carencia de una vacuna contra esta enfermedad hace necesario la búsqueda de nuevas alternativas terapéuticas para solucionar este problema de salud pública (WHO, 2010).

Dentro de las alternativas como nuevas fuentes terapéutica para el tratamiento de la leishmaniasis se ha recurrido a la obtención y evaluación de moléculas de origen natural y sintético. En el campo de la síntesis química se han hecho investigaciones como la obtención de derivados quinolínicos a partir de quinaldina e hidroxiquinaldina y algunos aldehídos aromáticos, así como la síntesis y caracterización de quinolinas unidas con carbenos $\mathrm{N}$-heterocíclicos que han mostrado una promisoria actividad leishmanicida (Sanchez et al., 2014).

Muchos productos naturales, especialmente las plantas representan un alto potencial terapéutico en el control y tratamiento de enfermedades. La familia Rutaceae constituye una fuente promisoria de metabolitos activos con un alto y diverso potencial para el tratamiento de muchas enfermedades, destacándose el estudio de la especie Galipea longiflora donde se evaluaron los extractos crudos y los alcaloides quinolínicos obtenidos de hoja y corteza contra promastigotes de: $L$. amazonensis, $L$. braziliensis, $L$. donovani y $L$. chagasi mostrando una buena actividad con valores de $I_{50}$ entre $76,5-86,4 \mu \mathrm{g} / \mathrm{mL}$ para el extracto crudo de corteza y 52,7 - 55,6 para el extracto crudo de hojas. Los alcaloides aislados a partir de estos extractos mostraron IC 50 entre $20,7-26,4 \mu \mathrm{g} / \mathrm{mL}$ (Gimenez et al., 2005). De la especie G. panamensis se aislaron 3 metabolitos secundarios tipo cumarinas denominados: $7-\left\{\left[\left(2 R^{\star}\right)-3,3\right.\right.$ - dimetiloxiran-2-il]metoxi $\}$ 8-[(2 $\left.R^{\star}, 3 R^{\star}\right)$-3-isopropeniloxiran-2-il]-2H-cromen-2-ona, 7-metoxi-8-(4-metil- 3-furil)-2H-cromen-2-ona y febalosina los cuales presentaron actividad leishmanicida con un $\mathrm{EC}_{50}$ de 9.9, 10.5 y $14.1 \mu \mathrm{g} / \mathrm{mL}$, respectivamente (Arango et al., 2010).

Algunos compuestos aislados de esta familia presentan reportes de actividad antimalárica y antioxidante (Macías et al., 2011; Cardona et al., 2013; Montaño et al., 2011; Guzman et al., 2013), efectos causados probablemente por compuestos de tipo alcaloidal con nucleo quinilínico (Patiño, 2011), furoquinolínico (Coy et al., 2013; Michael, 2008), indolopiridoquinazolínicos (Januario et al., 2009) y acridónicos (Ngoumfo et al., 2010); flavonoides tipo flavonas (Barros et al., 2007), cumarinas con actividad leishmanicida (Arango et al., 2010), así como actividad citotóxica antiproliferativa y antiinflamatoria (Macías et al., 2011). De la especie G. panamensis se aislaron 3 metabolitos secundarios tipo cumarinas denominados: $7-\left\{\left[\left(2 R^{\star}\right)-3,3-\right.\right.$ dimetiloxiran-2-il]metoxi\}-8-[(2 $\left.R^{\star}, 3 R^{\star}\right)$-3-isopropeniloxiran-2-il]-2H-cromen-2-ona, 7-metoxi-8-(4-metil- 3furil)-2 $\mathrm{H}$-cromen-2-ona y febalosina los cuales presentaros actividad leishmanicida con un $\mathrm{EC}_{50}$ de 9.9, 10.5 y $14.1 \mu \mathrm{g} / \mathrm{mL}$, respectivamente (Arango et al., 2010). El género Esenbeckia es uno de los más representativos de la familia Rutaceae por su gran aplicabilidad industrial y su uso tradicional en el tratamiento de algunas enfermedades, este representa una importante información etnobotánica de la cual derivan estudios fitoquímicos que demuestran la calidad de metabolitos secundarios que estas producen como alcaloides, cumarinas, flavonoides, triterpenos, lignanos (Coy et al., 2007; Cuca et al., 2011).

Basado en estos antecedentes se plantea la posibilidad de encontrar fracciones y metabolitos activos sobre amastigotes intracelulares de $L$. (V) panamensis; para ello se realizó el estudio fitoquímico de hojas, corteza y madera de la especie Esenbeckia litoralis perteneciente a la familia Rutaceae. En este trabajo se determinó la actividad leishmanicida así como la citotoxicidad en células U937 de fracciones de hojas, de corteza y de un compuesto puro. 


\title{
MATERIALES Y MÉTODOS
}

\author{
Material vegetal
}

La muestra de la especie Esenbeckia litoralis, fue colectada en el año 2013 en el municipio de San Juan de Nepomuceno, departamento de Bolívar, en las siguientes coordenadas geográficas: 9 $55^{\prime}$ 15,9" N; $75^{\circ} 05^{\prime}$ 39.3" W, del cual reposa una muestra en el Herbario de la Universidad de Córdoba bajo el número de colección HUC-005109.

\section{Obtención de extractos}

Las hojas, corteza y madera del material vegetal de la especie una vez seco y molido, se sometieron a extracción por percolación con etanol al $96 \%$ a temperatura ambiente. El solvente fue retirado a presión reducida obteniéndose así los correspondientes extractos etanólicos.

\section{Fraccionamiento y purificación de compuestos de Esenbeckia litoralis}

Los extractos etanólicos de hojas, corteza de Esenbeckia litoralis fueron fraccionados por reparto utilizando solventes de polaridad creciente: Benzina de petróleo (BDP), Cloroformo $\left(\mathrm{CHCl}_{3}\right)$, Acetato de etilo (AcOEt) y Agua $\left(\mathrm{H}_{2} \mathrm{O}\right)$.

\section{Fraccionamiento del extracto clorofórmico de hojas}

Se tomaron 15.6 gramos y se sometió a fraccionamiento por Cromatografía en Columna (CC) soportado en sílica gel, e iniciando el proceso de elusión con tolueno y aumentando la polaridad hasta $\mathrm{CHCl}_{3}$, obteniéndose 25 fracciones. Las fracciones 8,9 y 10 fueron reunidas según el perfil cromatográfico mostrado $(3,96 \mathrm{~g})$ y se sometieron a CC eluyendo con $\mathrm{CHCl}_{3}$, colectándose 7 nuevas fracciones desde A-1 hasta A-7. La fracción A-5 se sometió a CC repetitiva eluyendo con $\mathrm{CHCl}_{3}: \mathrm{MeOH}-98: 2$, así se aisló una sustancia pura denominada compuesto $1(34 \mathrm{mg})$.

\section{Fraccionamiento del extracto clorofórmico de corteza}

A partir de este extracto se tomaron 30,1 gramos y se sometió a fraccionamiento por Cromatografía en Columna (CC) soportado en sílica gel, e iniciando el proceso de elusión con BDP:diclorometano $\left(\mathrm{CH}_{2} \mathrm{Cl}_{2}\right)-$ 1:1 y aumentando la polaridad hasta $\mathrm{CH}_{2} \mathrm{Cl}_{2}: \mathrm{MeOH}-9: 1$, obteniéndose 26 fracciones (1 hasta 26) según el perfil cromatográfico mostrado. La fracción $18(765 \mathrm{mg})$ se sometió a CC eluyendo con Hex: $\mathrm{CH}_{2} \mathrm{Cl}_{2}-1: 1$ y aumentando la polaridad en forma creciente, colectándose 20 nuevas fracciones desde B-1 hasta B-20. De la fracción B-5 se aisló un sólido color naranja denominada compuesto $2(360 \mathrm{mg})$. La fracción B-6 $(210 \mathrm{mg})$ fue sometida a $\mathrm{CC}$ eluyendo con $\mathrm{CH}_{2} \mathrm{Cl}_{2}$ puro y aumentando la polaridad hasta $\mathrm{CH}_{2} \mathrm{Cl}_{2}: \mathrm{MeOH}$ - 95:5, obteniéndose 9 fracciones (B-6-1 hasta B-6-9). La fracción B-6-4 fue sometida a Cromatografía en Capa Delgada Preparativa (CCDP) eluyendo con $\mathrm{CH}_{2} \mathrm{Cl}_{2}: \mathrm{MeOH}-95: 5$, de la cual se obtuvieron cristales amarillos, con un peso de $11.6 \mathrm{mg}$ denominado compuesto 3 . Las fracciones B-7 y B-8 fueron reunidas (218 mg), sometidas a $\mathrm{CC}$ y eluidas con $\mathrm{CHCl}_{3}: \mathrm{MeOH}$ - 9:1, colectándose 50 fracciones reunidas posteriormente en 5 nuevas fracciones (B-7'-1 hasta B-7'-5) según el análisis por CCD. El perfil por CCD de la fracción B-7'4 , permitió denominar una nueva sustancia como compuesto 4 (10,3 mg).

La fracción A-7 (1,6 g) fue sometida a Cromatografía en Columna (133 g) eluyendo con $\mathrm{CHCl}_{3}$ puro y aumentando la polaridad hasta $\mathrm{CH}_{2} \mathrm{Cl}_{2}: \mathrm{MeOH}-95: 5$, colectándose 91 fracciones posteriormente reunidas en 8 fracciones (A-7-1 hasta A-7-8) por análisis en CCD. Las fracciones A-7-4 y A-7-5 fueron reunidas y sometidas a Cromatografía en Capa Delgada Preparativa (CCDP) eluyendo con $\mathrm{CH}_{2} \mathrm{Cl}_{2}: \mathrm{MeOH}-95: 5$, de la cual se obtuvieron cristales amarillos $(17,0 \mathrm{mg})$, mediante el análisis por CCD fueron denominados como compuesto 5 .

\section{Fraccionamiento del extracto etanólico de madera}

38 gramos de este extracto fueron sometidos a $\mathrm{CC}$ soportados sobre sílica gel, eluyendo con $\mathrm{CH}_{2} \mathrm{Cl}_{2}$ puro y aumentando su polaridad hasta $\mathrm{CHCl}_{3}$, las cuales fueron reunidas en 20 fracciones desde 1 hasta 20 . Teniendo en cuenta el análisis en CCD, las características de las manchas y el peso, la fracción 6 (791 mg) se sometió a un análisis por $\mathrm{CC}$ con sílica gel, eluyendo con $\mathrm{CHCl}_{3}$ y aumentando la polaridad hasta $\mathrm{CHCl}_{3}: \mathrm{CH}_{3} \mathrm{OH}-98: 2$ obteniéndose 93 fracciones $(15 \mathrm{ml} \mathrm{c} / \mathrm{u})$, posteriormente reunidas en 6 nuevas fracciones (B-1 hasta B-6) de acuerdo al estudio por CCD. La fracción B-3 (49 mg) fue sometida a CCDP eluyendo con $\mathrm{CHCl}_{3}$ por triplicado. De esta separación se obtuvieron 4 sustancias, de los cuales el mayoritario $(30 \mathrm{mg}$ ) se designó compuesto 6 . Las fracciones B-8, B-9 y B-10 fueron reunidas (566 mg) y 
solubilizadas en $\mathrm{CH}_{2} \mathrm{Cl}_{2}$, generando dos fases, una porción insoluble, la cual se separó y posteriormente se solubilizó en metanol y fue denominado compuesto $7(11,3 \mathrm{mg})$ según el perfil presentado en el análisis por CCD.

\section{Identificación de sustancias}

Los compuestos purificados fueron identificados utilizando las técnicas espectroscópicas IR, UV, RMN- ${ }^{1} \mathrm{H}$, $\mathrm{RMN}-{ }^{13} \mathrm{C}$, experimentos bidimensionales (COSY, HMQC, HMBC) y Cromatografía de gases acoplada a espectrometría de masa - CG-EM, y por comparación con datos reportados en la literatura.

\section{Ensayo de citotoxicidad en células U937 y evaluación de la actividad leishmanicida}

Para la evaluación de la actividad citotóxica se realizaron 6 diluciones dobles seriadas preparadas a partir de una solución madre de $200 \mu \mathrm{g} / \mathrm{ml}$ en dimetilsulfóxido (DMSO) (Sigma-Aldrich, St Louis MO, USA, obteniéndose seis soluciones (diluciones dobles seriadas) de concentración equivalente a 200,0 - 100,0 $50,0-25,0-12,5$ y $6,25 \mu \mathrm{g} / \mathrm{ml}$ para los extractos y para el compuesto.

Para la evaluación de la actividad leishmanicida inicialmente se preparó una solución a $20 \mu \mathrm{g} / \mathrm{ml}$. Y luego, para los compuestos con alguna actividad a esta concentración se prepararon diluciones cuádruples seriadas a partir de 100,0 - 25,0 - 6,25 y 1,562 $\mathrm{gg} / \mathrm{ml}$. Para la anfotericina B se prepararon cuatro soluciones a partir de $1,0 \mu \mathrm{g} / \mathrm{ml}$. Todas las soluciones se prepararon en DMSO al $0.5 \%$ y medio RPMI 1640 (Gibco, Thermo Scientific Inc., Waltham, MA, USA) completo, es decir, suplementado con $10 \%$ de suero fetal bovino (SFB) (Gibco) y $1 \%$ de antibióticos (penicilina-estreptomicina) (Sigma).

En los ensayos de actividad leishmanicida in vitro se utilizó la cepa de de L. (V) panamensis (UA140-pIR(-)eGFP) cultivados como promastigotes en medio bifásico NNN modificado (Novy-MacNeil-Nicolle), empleando como fase sólida agar sangre (Merck Millipore Corporation, Medellín, Colombia) fase líquida solución salina de buffer fosfato (PBS) más glucosa, $\mathrm{pH} 6.9$ e incubados a 26으 C. Los promastigotes se usaron posteriormente para infectar los macrófagos U937 y obtener amastigotes intracelulares (ver abajo). Con el fin de asegurar una mayor infección de los macrófagos in vitro, la cepa de $L$. (V) panamensis se mantiene en hámster (Mesocricetus auratus) que han sido infectados experimentalmente. Previamente a la infección in vitro de las células, se hizo un aspirado de la lesión del hámster con solución de fosfatos (PBS) y aguja \#26; luego, la muestra aspirada se cultivó en medio de cultivo NNN e incubados a una temperatura de $27^{\circ} \mathrm{C}$ hasta obtener promastigotes.

Monocitos humanos U937 (CRL-1593-2 ${ }^{\mathrm{TM}}$ ), adquirida del ATCC (American Type Cell Collection) se mantuvieron cultivadas en suspensión en medio RPMI-1640 completo (ver arriba) e incubación a 37드. con $5 \%$ de $\mathrm{CO}_{2}$.

\section{Actividad citotóxica sobre células U937.}

La citotoxicidad se evaluó sobre las células U-937 empleando el micrométodo enzimático con bromuro de 3(4,5-dimetiltiazol-2-il)-2,5-difeniltetrazolio (MTT). Las células en fase exponencial de crecimiento se ajustaron a una concentración de 100.000 células/ml de medio RPMI-1640 suplementado con SFB y antibióticos, como se describió arriba. En cada pozo de un plato para cultivo celular de 96 pozos (Falcon, Fisher Scientific, Thermo Scientific Inc, Waltham, MA, USA) se depositaron $100 \mu$ lde células. Luego se adiciono en cada pozo 100 ul de cada una de las correspondientes concentraciones para el respectivo compuesto. Como control negativo (viabilidad o no citotoxicidad) se usaron células en medio RPMI completo y mantenidas bajo las mismas condiciones de incubación pero en ausencia de cualquier extracto o del compuesto; como control de citotoxicidad se utilizaron células expuestas a anfotericina B.

Las células en presencia de las diferentes soluciones de los extractos y del compuesto, así como los controles se incubaron a $37^{\circ} \mathrm{C}$ en una atmósfera al $5 \%$ de $\mathrm{CO}_{2}$ durante 72 horas. Transcurrido el período de incubación, el efecto de los extractos y del compuesto sobre las células se determinó midiendo la actividad de la enzima mitocondrial succinato deshidrogenasa. Para ello, se adicionó, $10 \mu \mathrm{l} /$ pozo de una solución de MTT con concentración $5 \mu \mathrm{g} / \mathrm{ml}$ (Sigma) y se incubaron los platos a $37^{\circ} \mathrm{C}$ durante 3 horas. Pasado este período de incubación se adicionaron $100 \mu \mathrm{l} /$ pozo de una solución de 50\% de isopropanol (Merck Millipore) y $10 \%$ de dodecil sulfato de sodio (SDS) (Merck Millipore) para solubilizar los cristales de formazán (Insuasty et al, 2015). Los platos se incubaron durante otros 30 minutos y la producción de formazán (que es proporcional al porcentaje de células viables) se midió en un lector de microplacas (Benchmark Bio-Rad Hercules CA, USA) a una densidad óptica de $570 \mathrm{~nm}$. 
La citotoxicidad se determinó de acuerdo al porcentaje de disminución de la viabilidad y por ende, de la disminución en la cantidad de células obtenidas para cada extracto o compuesto y la anfotericina B según las densidades ópticas (D.O) obtenidas en cada condición experimental. La disminución de la viabilidad celular denominada inhibición del crecimiento celular, se calculó utilizando los valores de D.O para cada condición, es decir, extracto, compuesto o control a la concentración evaluada, usando la siguiente ecuación: \% Viabilidad $=[D .0$ células expuestas al compuesto o extracto o control $\div$ D.O células no expuestas] $\times$ 100). Los valores de D.O obtenidas para las células en ausencia de compuestos o extractos corresponde al $100 \%$ de viabilidad o células vivas. Lego, con los porcentajes de viabilidad se calculó el porcentaje de mortalidad, que corresponde a 100 - \% viabilidad. Por último, con los porcentajes de mortalidad se calculó la concentración letal $50\left(\mathrm{CL}_{50}\right)$ calculada por el método de análisis dosis respuesta, Probit (Finney, 1971) usando el programa estadístico SAS Data Analysis (SAS Institute Cary NC, USA). LoS ensayos se realizaron dos veces con tres réplicas para cada concentración evaluada. La citotoxicidad de cada extracto o compuesto se clasificó según los valores de $C_{50}$ usando una escala propia: citotoxicidad alta $\mathrm{CL}_{50}<50 \mu \mathrm{g} / \mathrm{mL}$; citotoxicidad moderada: $\mathrm{CL}_{50}>50$ a $<200 \mu \mathrm{g} / \mathrm{mL}$ y citotoxicidad baja: $\mathrm{CL}_{50}>200$ $\mu \mathrm{g} / \mathrm{mL}$.

\section{Actividad sobre L. (V) panamensis}

La actividad de los extractos y el compuesto se evaluó sobre amastigotes intracelulares obtenidos luego de la infección in vitro de macrófagos U937 diferenciados con miristato acetato de forbol (PMA del inglés phorbol myristate acetate) (Sigma). Para ello, las células U937 mantenidas en cultivo en suspensión se centrifugaron a $1.500 \mathrm{rpm}$ durante 10 minutos y luego de descartar el sobrenadante, el botón de células se resuspendió a una concentración de $1 \times 10^{5}$ células $/ \mathrm{ml}$ de medio RPMl 1640 al 10\% SFB con $0.1 \mu \mathrm{g} / \mathrm{ml}$ de PMA. En cada pozo de una placa para cultivo celular de 24 pozos se dispersó 1 ml de la suspensión de células y se incubaron a una temperatura de $37^{\circ} \mathrm{C}$ con una atmosfera al $5 \% \mathrm{CO}_{2}$. Transcurridas 48 horas de incubación las células se infectaron con promastigotes en fase estacionaria de crecimiento en una proporción 15:1 parásito: célula. Los platos se incubaron a $34^{\circ} \mathrm{C}$ en un atmosfera al $5 \%$ de $\mathrm{CO}_{2}$ durante 2 horas. Posteriormente se realizaron dos lavados con PBS para eliminar los parásitos libres, se adicionó un $\mathrm{ml}$ de medio RPMI 1640 completo y las células se incubaron nuevamente durante 24 horas. Pasado este tiempo, las células infectadas se expusieron a cada una de las concentraciones de los extractos y del compuesto durante 72 horas. Como control de infección se usaron células infectadas y cultivadas en ausencia de los extractos y del compuesto y como control de efectividad se empleó la anfotericina B. Luego de 72 horas de incubación a $34^{\circ} \mathrm{C}$ en un atmosfera al $5 \%$ de $\mathrm{CO}_{2}$ las células se removieron cuidadosamente del fondo del plato utilizando el émbolo de una jeringa y se analizaron en un citómetro de flujo leyendo a 488 nm de excitación y 525 nm de emisión con un láser de Argón (Galeano et al., 2011 ). Cada concentración de los extractos, del compuesto y de los controles se evaluó por triplicado en dos experimentos diferentes. La actividad anti-Leishmania se determinó de acuerdo a la carga parasitaria, es decir, la cantidad de parásitos en las células infectadas obtenidas para cada concentración de cada extracto, compuesto y anfotericina $\mathrm{B}$ según el número de eventos positivos para la flurescencia verde usando diagrama de puntos e histogramas según la intensidad media de flurescencia (IFM) (Pulido et al, 2012).

La disminución de la carga parasitaria, denominada inhibición de la infección, se calculó utilizando las los valores de IFM para cada condición, es decir, extracto, compuesto o control a la concentración evaluada, usando la siguiente ecuación: \% Infección = [IFMI células infectadas y expuestas al compuesto o extracto o control $\div$ IFM células infectadas y no expuestas] × 100). Los valores de IFM obtenidas para las células infectadas en ausencia de compuestos o extractos corresponde al $100 \%$ de parásitos. A su vez, el porcentaje de inhibición de la infección corresponde a 100 - \% infección

Para los compuestos que mostraron alguna inhibición a $20 \mu \mathrm{g} / \mathrm{ml}$, se determinó también la Concentración Efectiva $50\left(\mathrm{EC}_{50}\right)$ calculada por el método Probit (Finney, 1971). Al igual que la citotoxicidad, la actividad anti-leishmania se clasificó según los valores de $C_{50}$ así: actividad alta: $C_{50}<25 \mu \mathrm{g} / \mathrm{mL}$; actividad moderada: $\mathrm{CL}_{50}>25 \mathrm{a}<50 \mu \mathrm{g} / \mathrm{mL}$ y actividad baja: $\mathrm{CL}_{50}>50 \mu \mathrm{g} / \mathrm{mL}$. Finalmente, la actividad citotóxica se correlacionó con la actividad anti-Leishmania calculando el Índice de Selectividad (IS) con la siguiente ecuación: $I S=C L_{50} \div \mathrm{CE}_{50}$.

\section{RESULTADOS Y DISCUSIÓN}

\section{Compuestos aislados}

De la especie $E$. litoralis se purificaron siete compuestos (Fig 1): cinco alcaloides furoquinolínicos, uno acridónico y un flavonoide. De las hojas se aisló la Flindersiamina (1), de la corteza se aislaron 1-hidroxi-3metoxi- $N$-metilacridona (2), Maculosidina (3), Kokusaginina (4) y Maculina (5) y de la madera se purificaron 
la Dictamina (6) y un flavonoide denominado Gardenia B (7), la asignación de las estructuras se realizó con base en el análisis de los datos espectroscópicos.

Flindersiamina (1): Sólido amarillo; p.f. $185-186^{\circ} \mathrm{C}$; IR: (KBr) $\lambda_{\max } 3133 \mathrm{~cm}^{-1}$ (C-H aromático), 2956-2813 cm1 (C-H, alifáticos), $1625 \mathrm{~cm}^{-1}(\mathrm{C}=\mathrm{C}), 1369 \mathrm{~cm}^{-1}(\mathrm{C}-\mathrm{O}-\mathrm{C})$; EM m/z $273\left(\mathrm{M}^{+}\right)$; RMN-1 ${ }^{-1} \mathrm{H}\left(500 \mathrm{MHz}, \mathrm{CDCl}_{3}\right) ; \delta_{\mathrm{H}}$ : $7.55(\mathrm{~d}, \mathrm{~J}=2.8 \mathrm{~Hz}, 1 \mathrm{H})$ y $\delta_{\mathrm{H}}: 7.00(\mathrm{~d}, \mathrm{~J}=2.8,1 \mathrm{H})$, protones de anillo furánico; un protón aromático en $\delta_{\mathrm{H}}: 7.22$ $(\mathrm{s}, 1 \mathrm{H}) \mathrm{ppm}$; $\delta_{\mathrm{H}}: 6.04\left(\mathrm{~s}, 2 \mathrm{H}\right.$ - metilendioxi) y protones en $\delta_{\mathrm{H}}: 4.35(\mathrm{~s}, 3 \mathrm{H})$ y $\delta_{\mathrm{H}}: 4.25(\mathrm{~s}, 3 \mathrm{H})$ de grupos metoxilos. RMN-13C (125 MHz, CDCl 3 ) y DEPT-135: ocho carbonos cuaternarios en $\delta_{c}$ : 163.0, 156.0, 146.7, 138.0, 137.6, 135.9, 114.9 y 102.2 ppm; tres $\mathrm{CH}$ en $\delta_{c}: 142.4$, 104.4 y 100.1 ppm; un $\mathrm{CH}_{2}$ en $\delta_{c}$ : 101.5 ppm, dos $\mathrm{CH}_{3}$ en $\delta_{\mathrm{c}}: 60.6$ y $58.8\left(-\mathrm{OCH}_{3}\right)$.

1-hidroxi-3-metoxi-N-metilacridona (2): Sólido anaranjado; p.f. $169-170{ }^{\circ} \mathrm{C}$; IR: (KBr) $\lambda_{\max } 3345(\mathrm{OH}), 1639$, 1592 y $1556\left(\mathrm{C}=\mathrm{C}\right.$, aromáticos) y $1643(\mathrm{C}=\mathrm{O}) \mathrm{cm}^{-1}$; EM (IE) m/z: $255\left(\mathrm{M}^{+}\right) ; \mathrm{RMN}^{-1} \mathrm{H}\left(500 \mathrm{MHz}, \mathrm{CDCl}_{3}\right) \delta_{\mathrm{H}}$ : $3.85\left(\mathrm{~s}, 3 \mathrm{H},-\mathrm{OCH}_{3}\right) ; \delta_{\mathrm{H}}: 2.70\left(\mathrm{~s}, 3 \mathrm{H},-\mathrm{NCH}_{3}\right) ; \delta_{\mathrm{H}}: 6.20(\mathrm{~d}, \mathrm{~J}=2 \mathrm{~Hz}, 1 \mathrm{H})$ y $\delta_{\mathrm{H}}: 6.19(\mathrm{~d}, \mathrm{~J}=2 \mathrm{~Hz}, 1 \mathrm{H})$ protones aromáticos en posición meta; $\delta_{\mathrm{H}}: 7.23(\mathrm{t}, \mathrm{J}=7.60 \mathrm{~Hz}, 1 \mathrm{H}) ; \delta_{\mathrm{H}}: 7.40\left(\mathrm{~d}, \mathrm{~J}=8.7 \mathrm{~Hz}, 1 \mathrm{H}\right.$ ); $\delta_{\mathrm{H}}: 7.66$ (ddd, J=7.6, $7.8 \mathrm{~Hz}, 1 \mathrm{H})$ y $\delta_{H}: 8.40(\mathrm{dd}, \mathrm{J}=1.6,8.0 \mathrm{~Hz}, 1 \mathrm{H})$ de un anillo aromático disustituido; $\delta_{\mathrm{H}}: 14.80(\mathrm{~s}, 1 \mathrm{H})$ correspondiente a un $\mathrm{OH}$ fenólico. $\mathrm{RMN}-{ }^{13} \mathrm{C}\left(125 \mathrm{MHz}, \mathrm{CDCl}_{3}\right)$ y DEPT-135: siete carbonos cuaternarios en $\delta_{c}: 180.6(\mathrm{C}=\mathrm{O}), 166.0,165.8,144.2,142.3,120.9,105.2 \mathrm{ppm}$; seis $\mathrm{CH}$ en $\delta_{c}: 134.0,126.6,121.3,114.5$, 94.1, 89.8 ppm; un $\mathrm{CH}_{3}$ en $\delta_{\mathrm{c}}: 55.5\left(-\mathrm{OCH}_{3}\right)$ y $\delta_{\mathrm{c}}: 34.0\left(-\mathrm{NCH}_{3}\right) \mathrm{ppm}$.

Maculosidina (3): Sólido amarillo; IR: (KBr) $\lambda \max 3131 \mathrm{~cm}^{-1}\left(\mathrm{C}-\mathrm{H}\right.$, aromáticos), 2998 y $2954 \mathrm{~cm}^{-1}(\mathrm{C}-\mathrm{H}$, alifáticos), $1625 \mathrm{~cm}^{-1}(\mathrm{C}=\mathrm{C}), 1483 \mathrm{~cm}^{-1}$ (C-C), $1258 \mathrm{~cm}^{-1}$ (C-O-C) ; EM m/z $259\left(\mathrm{M}^{+}\right)$; RMN-1 ${ }^{-1}(500 \mathrm{MHz}$, $\left.\mathrm{CDCl}_{3}\right) ; \delta_{\mathrm{H}}: 7.43(\mathrm{~s}, 1 \mathrm{H})$ y $\delta_{\mathrm{H}}: 7.31(\mathrm{~s}, 1 \mathrm{H})$, protones aromáticos en anillo tetrasustituído; $\delta_{\mathrm{H}}: 7.54(\mathrm{~d}, \mathrm{~J}=2.1$ $\mathrm{Hz}, 1 \mathrm{H})$ y $\delta_{H}: 7.00(\mathrm{~d}, \mathrm{~J}=2.1 \mathrm{~Hz}, 1 \mathrm{H})$ protones característicos de un anillo furánico; $\delta_{H}: 4.40(\mathrm{~s}, 3 \mathrm{H}), \delta_{H}: 4.02$ $(\mathrm{s}, 3 \mathrm{H})$ y $\delta_{\mathrm{H}}: 4.01(\mathrm{~s}, 3 \mathrm{H})$ protones de grupos metoxilos. RMN- ${ }^{13} \mathrm{C}\left(125 \mathrm{MHz}, \mathrm{CDCl}_{3}\right)$ y DEPT-135, 90 y 45 : siete carbonos cuaternarios en $\delta_{c}: 163.1,155.4,152.6,147.7,142.6,112.9$ y 102.2 ppm; cuatro $\mathrm{CH}$ en $\delta_{c}$ : 142.4, 106.7, 104.6, 100.2 ppm; tres $\mathrm{CH}_{3}$ en $\delta$ c: $58.8,56.0$ y 55.9 ppm característicos de metoxilos.

Kokusaginina (4): Sólido amarillo; IR: $(\mathrm{KBr}) \lambda_{\max } 3142 \mathrm{~cm}^{-1}\left(\mathrm{C}-\mathrm{H}\right.$, aromáticos), 2954 y $2915 \mathrm{~cm}^{-1}(\mathrm{C}-\mathrm{H}$, alifáticos), 1624, 1590, $1508 \mathrm{~cm}^{-1}$ (C=C), $1483 \mathrm{~cm}^{-1}$ (C-C), $1260 \mathrm{~cm}^{-1}$ (C-O-C) ; EM m/z $259\left(\mathrm{M}^{+}\right)$; RMN-1 ${ }^{-1}$ $\left(500 \mathrm{MHz}, \mathrm{CDCl}_{3}\right) ; \delta_{\mathrm{H}}: 7.31(\mathrm{~s}, 1 \mathrm{H})$ y $\delta_{\mathrm{H}}: 7.28(\mathrm{~s}, 1 \mathrm{H})$, protones aromáticos en anillo tetrasustituído; $\delta_{\mathrm{H}}: 7.48$ $(\mathrm{d}, \mathrm{J}=2.7 \mathrm{~Hz}, 1 \mathrm{H})$ y $\delta_{\mathrm{H}}: 6.90(\mathrm{~d}, \mathrm{~J}=2.7 \mathrm{~Hz}, 1 \mathrm{H})$ protones característicos de un anillo furánico; $\delta_{\mathrm{H}}: 4.30(\mathrm{~s}, 3 \mathrm{H})$, $\delta_{\mathrm{H}}: 4.01(\mathrm{~s}, 3 \mathrm{H})$ y $\delta_{\mathrm{H}}: 3.98(\mathrm{~s}, 3 \mathrm{H})$ protones de grupos metoxilos. RMN- ${ }^{13} \mathrm{C}\left(125 \mathrm{MHz}, \mathrm{CDCl}_{3}\right)$ y DEPT-135, 90 y 45 : siete carbonos cuaternarios en $\delta_{c}$ : $162.9,155.3,152.5,147.6,142.4,112.8$ y 102.0 ppm; cuatro $\mathrm{CH}$ en $\delta_{c}: 142.2,106.5,104.6,100.2$ ppm; tres $\mathrm{CH}_{3}$ en $\delta_{c}: 58.7,56.0$ y 55.9 ppm característicos de metoxilos.

Maculina (5): Sólido amarillo; p.f. 200-201 을 $\mathrm{IR}$ : (KBr) $\lambda_{\max } 3125$ y $3100 \mathrm{~cm}^{-1}\left(\mathrm{C}-\mathrm{H}\right.$ aromáticos), $2925 \mathrm{~cm}^{-1}$ (C-H, alifáticos), $1619 \mathrm{~cm}^{-1}$ (C-N), $1466 \mathrm{~cm}^{-1}$ (C-C), $1368 \mathrm{~cm}^{-1}$ (C-O-C) ; EM m/z $243\left(\mathrm{M}^{+}\right)$; RMN-1H (500 $\left.\mathrm{MHz}, \mathrm{CDCl}_{3}\right) ; \delta_{\mathrm{H}}: 7.55(\mathrm{~d}, \mathrm{~J}=2.7 \mathrm{~Hz}, 1 \mathrm{H})$ y $\delta_{\mathrm{H}}: 7.02(\mathrm{~d}, \mathrm{~J}=2.7,1 \mathrm{H})$, protones de anillo furánico; protones aromáticos en anillo tetrasustituído en $\delta_{\mathrm{H}}: 7.49(\mathrm{~s}, 1 \mathrm{H})$ y $\delta_{\mathrm{H}}: 7.27(\mathrm{~s}, 1 \mathrm{H}) \mathrm{ppm}, \delta_{\mathrm{H}}: 6.08(\mathrm{~s}, 1 \mathrm{H}$ - metilendioxi) y $\delta_{\mathrm{H}}: 4.40$ (s, $3 \mathrm{H}$ - metoxilos). RMN- ${ }^{13} \mathrm{C}\left(125 \mathrm{MHz}, \mathrm{CDCl}_{3}\right)$ y DEPT-135: siete carbonos cuaternarios en $\delta_{\mathrm{c}}$ : $163.1,156.0,151.0,146.0,144.0,114.0$ y 101.6 ppm; cuatro $\mathrm{CH}$ en $\delta_{c}$ : $142.6,104.5,104.4,98.0$ ppm; un $\mathrm{CH}_{2}$ en $\delta_{c}: 101.6 \mathrm{ppm}$, un $\mathrm{CH}_{3}$ en $\delta_{c}: 58.9\left(-\mathrm{OCH}_{3}\right)$.

Dictamina (6): Sólido amarillo; p.f. $130-131^{\circ} \mathrm{C}$; IR: (KBr) $\lambda_{\max } 3108 \mathrm{~cm}^{-1}$ (C-H aromáticos), 2929, $2856 \mathrm{~cm}^{-1}$ (C-H, alifáticos), $1372 \mathrm{~cm}^{-1}$ (C-O); EM m/z $199\left(\mathrm{M}^{+}\right)$; RMN-1 ${ }^{-1}\left(500 \mathrm{MHz}, \mathrm{CDCl}_{3}\right.$ ); $\delta_{\mathrm{H}}: 7.17$ (ddd, J=8.0, 1.2 $\mathrm{Hz}, 1 \mathrm{H}$ ), $\delta_{\mathrm{H}}: 7.45$ (ddd, J=8.0, $1.3 \mathrm{~Hz}, 1 \mathrm{H}$ ), $\delta_{\mathrm{H}}: 7.77$ (dd, J=8.5 Hz, 1H) y $\delta_{\mathrm{H}}: 8.02$ (dd, J=8.4, Hz, $1 \mathrm{H}$ ) protones aromáticos, $\delta_{H}: 7.36(\mathrm{~d}, \mathrm{~J}=2.8 \mathrm{~Hz}, 1 \mathrm{H})$ y $\delta_{\mathrm{H}}: 6.80(\mathrm{~d}, \mathrm{~J}=2.8 \mathrm{~Hz}, 1 \mathrm{H})$ protones de anillo furánico, $\delta_{\mathrm{H}}$ : $4.17\left(\mathrm{~s}, 3 \mathrm{H},-\mathrm{OCH}_{3}\right) \cdot \mathrm{RMN}^{13} \mathrm{C}\left(125 \mathrm{MHz}, \mathrm{CDCl}_{3}\right)$ y DEPT-135: cinco carbonos cuaternarios en $\delta_{\mathrm{c}}: 164.2$, $157.1,146.0,119.0$ y 103.7 ppm; seis $\mathrm{CH}$ en $\delta_{c}: 143.8,129.9,128.1,124.0,122.7$ y $105.1 \mathrm{ppm}$ y un $\mathrm{CH}_{3}$ en ठc: $59.3\left(-\mathrm{OCH}_{3}\right)$

Gardenina $B$ (7): Sólido blanco; IR: (KBr) $\lambda_{\max } 3389 \mathrm{~cm}^{-1}(\mathrm{O}-\mathrm{H}), 2929,2928 \mathrm{~cm}^{-1}\left(\mathrm{C}-\mathrm{H}\right.$, alifáticos), $1715 \mathrm{~cm}^{-1}$ (C-O); RMN-1 ${ }^{1} \mathrm{H}\left(500 \mathrm{MHz}, \mathrm{CDCl}_{3}\right) ; \delta_{\mathrm{H}}: 7.90$ (d, J=8.8 Hz, 2H) y $\delta_{\mathrm{H}}: 7.04$ (d, J=8.8 Hz, 2H) característicos de un anillo aromático 1,4 disustituído, $\delta_{\mathrm{H}}: 6.61(\mathrm{~s}, 1 \mathrm{H}), \delta_{\mathrm{H}}: 12.80(\mathrm{~s}, 1 \mathrm{H})$ específico para un protón de grupo $\mathrm{OH}, \delta_{H}: 4.12(\mathrm{~s}, 3 \mathrm{H}), \delta_{H}: 3.98(\mathrm{~s}, 3 \mathrm{H}), \delta_{H}: 3.96(\mathrm{~s}, 3 \mathrm{H})$ y $\delta_{H}: 3.90(\mathrm{~s}, 3 \mathrm{H})$ protones de grupos metoxilos. RMN${ }^{13} \mathrm{C}\left(125 \mathrm{MHz}, \mathrm{CDCl}_{3}\right)$ y DEPT-135: diez carbonos cuaternarios en $\delta_{\mathrm{c}}$ : $183.0,164.0,162.0,152.0,149.0$, $145.8,136.5,132.9,128.0$ y 123.4 ppm; cinco $\mathrm{CH}$ en $\delta_{c}$ : $128.0(2 \mathrm{C}), 114.0$ (2C) y 106.0 , y cuatro grupos $\mathrm{CH}_{3}$ en $\delta_{c}: 62.1, \delta_{c}: 61.7, \delta_{c}: 61.1$ y $\delta_{c}: 55.6$, característicos de grupos metoxilos. 
<smiles>COc1c2ccoc2nc2c(OC)c3c(cc12)OCO3</smiles>

1<smiles>CO/C=C/C1C=c2ccoc2=N[C@@H]1/C=C/OC</smiles>

4<smiles></smiles>

2<smiles>COc1c2cc3c(cc2nc2occc12)OCO3</smiles>

5<smiles>COc1cc(OC)c2nc3occc3c(OC)c2c1</smiles>

3<smiles>COc1c2ccccc2nc2occc12</smiles>

6<smiles>COc1ccc(-c2cc(=O)c3c(O)c(OC)c(OC)c(OC)c3o2)cc1</smiles>

Fig. 1. Compuestos aislados de Esenbeckia litoralis (Rutaceae).

El estudio fitoquímico de la especie E. litoralis permitió la obtención de 6 metabolitos secundarios de naturaleza alcaloidal donde 5 corresponden a alcaloides con núcleo furoquinolínico y uno con núcleo acridónico, todos ellos han sido aislados anteriormente de especies pertenecientes al género esenbeckia, pero los alcaloides Flindersiamina (1), 1-hidroxi-3-metoxi-N-metilacridona (2), Maculosidina (3), Kokusaginina (4) y Maculina (5) y la Dictamina se reportan por primera vez para esta especie, los compuestos fueron identificados por el análisis de los datos espectroscópicos y también por comparación con los valores de los mismos compuestos descritos en la literatura. El compuesto 7 aislado del extracto etanólico de madera conocido como Gardenina B es flavonoide con núcleo tipo flavona aislado de la especie E. litoralis el cual fue reportado por Torres en 2001 (Coy et al., 2013). Esta investigación muestra la diversidad de sustancias en las diferentes partes de la planta que presenta la mayor abundancia de alcaloides en el extracto de corteza. Este estudio permite proponer al compuesto 2 como compuesto líder para realizar modificaciones moleculares sobre la parte fenólica, con el fin de buscar mejores agentes leishmanicidas, ya que modificaciones hechas sobre este tipo de núcleo han mostrado mejorar el potencial leishmanicida, caso soportado en la síntesis de los compuestos 4-(6-nitro-benzotiazol-2-ilamino)-10Hacridin-9-ona y 1-(6-amino-benzothiazol-2-ilamino)-10H-acridin-9-ona que presentaron una buena actividad leishmanicida (Delmas et al., 2004).

\section{Potencial Leishmanicida}

La actividad in vitro de extractos de hojas y corteza de E. litoralis así como de uno de los metabolitos aislado de la corteza frente a amastigotes intracelulares de L. (V) panamensis, UA140-pIR(-)-eGFP y células U937 se resumen en la Tabla 1. Los compuestos puros 1, 3, 4, 5, 6 y 7 no se evaluaron por que se aislaron en pequeñas cantidades, las cuales fueron usadas para su identificación por técnicas espectroscópicas. Los datos representan el promedio \pm la desviación estándar de la Concentración Letal 50 (CL50) y la Concentración Efectiva $50\left(\mathrm{CE}_{50}\right)$ en $\mu \mathrm{g} / \mathrm{ml}$ y el Porcentaje de Inhibición a una concentración de $20 \mu \mathrm{g} / \mathrm{ml}$. NE: No evaluado. *Concentración evaluada: $0.5 \mu \mathrm{g} / \mathrm{ml}$. Según la escala aquí establecida, la mayoría de los extractos, tanto de corteza como de hojas mostraron citotoxicidad moderada, con valores de CL50 > 50 y $<$ $200 \mu \mathrm{g} / \mathrm{ml}$, con excepción del extracto en hexano de corteza y el extracto en acetato de etilo de hojas, que arrojaron citotoxicidad alta, con valores de $\mathrm{CL}_{50}<50 \mu \mathrm{g} / \mathrm{ml}$ y el extracto en cloroformo de corteza y compuesto 2 que no mostraron citotoxicidad (valores de $\mathrm{CL}_{50}>200 \mu \mathrm{g} / \mathrm{ml}$. Como se esperaba, la anfotericina B fue citotóxica $\left(\mathrm{CL}_{50}<50 \mu \mathrm{g} / \mathrm{ml}\right)$. 
Tabla 1. Actividad citotóxica y leishmanicida de los extractos de Esenbeckia litoralis (Rutaceae).

\begin{tabular}{|c|l|r|r|c|}
\hline Muestra & Extractos & \multicolumn{1}{|c|}{ CL50 } & \% Inhibición & CE $_{50}$ \\
\hline \multirow{4}{*}{ Corteza } & Etanol & $83.8 \pm 3.8$ & 0.0 & $\mathrm{NE}$ \\
\cline { 2 - 5 } & Hexano & $15.3 \pm 4.0$ & 0.0 & $\mathrm{NE}$ \\
\cline { 2 - 5 } & Cloroformo & $>200.0$ & 0.0 & $\mathrm{NE}$ \\
\cline { 2 - 5 } & Acetato de etilo & $64.9 \pm 10.6$ & $9.2 \pm 1.0$ & $78.26 \pm 3.91$ \\
\cline { 2 - 5 } & Compuesto 2 & $>200.0$ & $37.5 \pm 8.9$ & $24.8 \pm 5.88$ \\
\hline \multirow{4}{*}{ Hojas } & Etanol & $114.8 \pm 2.7$ & 0.0 & $\mathrm{NE}$ \\
\cline { 2 - 5 } & Cloroformo & $83.8 \pm 3.8$ & 0.0 & $\mathrm{NE}$ \\
\cline { 2 - 5 } & Acetato de etilo & $38.6 \pm 3.4$ & 0.0 & $\mathrm{NE}$ \\
\hline Control & Anfotericina B & $38.6 \pm 0.2$ & $62.7 \pm 1.4^{*}$ & $0.05 \pm 0.001$ \\
\hline
\end{tabular}

Por su parte, la mayoría de los extractos no mostraron actividad contra amastigotes intracelulares de $L$. (V) panamensis, evidenciada por la incapacidad de inhibir la carga parasitaria $(0 \%$ de inhibición a $20 \mu \mathrm{g} / \mathrm{ml})$. Solo extracto acetato de etilo mostro actividad pobre, inhibiendo el $9.2 \%$ de la carga parasitaria cuando las células infectadas se expusieron a una concentración de $20 \mu \mathrm{g} / \mathrm{ml}$. Este bajo porcentaje de inhibición se correlacionó con un valor de $\mathrm{CE}_{50}$ de $78.26 \pm 3.91 \mu \mathrm{g} / \mathrm{ml}$, el cual según la escala establecida lo clasifica en el grupo de actividad baja. Por el contrario, el compuesto 2 si mostró actividad contra L. (V) panamensis, siendo capaz de inhibir la carga parasitaria en un $37.2 \%$. Una $\mathrm{CE}_{50}<25 \mu \mathrm{g} / \mathrm{ml}$ determinada para el compuesto 2 permite clasificarlo como un compuesto con alta actividad contra la especie de Leishmania aquí evaluada. Por último, la anfotericina $\mathrm{B}$ que es uno de los medicamentos usados en el tratamiento de la leishmaniasis mostro ser altamente activo contra amastigotes intracelulares de $L$. (V) panamensis $(62.7 \%$ de inhibición a $0.5 \mu \mathrm{g} / \mathrm{ml}$ y $C_{50} 0.05 \pm 0.001 \mu \mathrm{g} / \mathrm{ml}$ ).

Los resultados anteriores evidencian el potencial leishmanicida del compuestos $\mathbf{2}$, dada la baja citotoxicidad comparado con el medicamento de referencia Anfotericina B y una adecuada actividad anti-Leishmania a bajas concentraciones. Estos resultados resaltan además la importancia de evaluar en el futuro los otros metabolitos aislados y que no se pudieron evaluar por la no disponibilidad de cantidad suficiente. Los resultados mostrados por el extracto en acetato de etilo de corteza sugiere la presencia de compuestos con capacidad inhibitoria contra el parásito de Leishmania probablemente flavonoides, cumarinas (Torres; 2001) y alcaloides (Coy et al., 2013), compuestos característicos del género y de esta especie que han presentado una diversidad de actividades biológicas; de esta forma se puede notar que los metabolitos antileishmánicos mayoritarios están presentes en los extractos con mayor polaridad como son el extracto en cloroformo y en acetato de etilo de corteza.

\section{CONCLUSIONES}

Del estudio fitoquímico de las diferentes partes de E. litoralis (Rutaceae), se aislaron siete compuestos con diversidad molecular: cinco alcaloides con nucleos furanoquinolínicos: Flindersiamina (1), Maculosidina (3), Kokusaginina (4), Maculina (5) y Dictamina (6), un alcaloide con núcleo tipo acridona: 1-hidroxi-3-metoxi- $N$ metilacridona (2) y un flavonoide tipo flavona: gardenina B (7).

La actividad in vitro del compuesto 2 y del extracto en acetato de etilo de corteza mostrada frente a $L$. (V) panamensis, para $E$. litoralis aporta bases para continuar estudios tendientes a disponer una molécula candidata que pueda convertirse en una alternativa para el tratamiento de la leishmaniasis.

\section{AGRADECIMIENTOS}

Los autores agradecen a la Universidad de Córdoba, por el apoyo financiero para el desarrollo de la presente investigación; a los profesores de la Universidad de Córdoba Heidy Saab Ramos y Rosalba Ruiz Vega quienes clasificaron el ejemplar y a la Universidad de Antioquía (convocatoria CIDEPRO-CIIEs).

\section{REFERENCIAS}

Alvar, J. y otros siete autores, WHO Leishmaniasis Control Team. Leishmaniasis worldwide and global estimates of its incidence, doi: 10.1371/journal.pone.0035671, PLoS One.;7(5):e35671(2012) 
Arango, V. y otros seis autores, Coumarins from Galipea panamensis and Their Activity against Leishmania panamensis, doi: 10.1021/np100146y, J. Nat. Prod: 73(5), 1012-1014 (2010)

Barros, B. A. y otros siete autores, Metabólitos secundários de Esenbeckia almawillia kaastra (Rutaceae), doi.org/10.1590/S0100-40422007000700017, Quim. Nova: 30 (7), 1589-1591 (2007)

Cardona, W., S. M. Robledo., B. A. Rojano., F. A. Guarin., D. L. Muñoz. y J. S. Vega, Actividad leishmanicida y antioxidante de extractos de Piper daniel-gonzalezii Trel. (Piperaceae), http://scielo.sld.cu/scielo.php?pid=S1028-47962013000200010\&script=sci_arttext; ISSN: 1028-4796, Rev. Cubana Plant. Med: 18 (2), 1-10 (2013)

Coy, C. A. y L. E. Cuca, Metabolitos secundarios aislados de los géneros Raputia y Esenbeckia (Rutaceae), http://dialnet.unirioja.es/descarga/articulo/4815070.pdf; ISSN: 0122-1701, Scientia et Technica: Año XIII (33), 337-338 (2007)

Coy, C. A., L. E. Cuca. y C. Quintero, Farmacognosia y farmacobotánica de especies pertenecientes a los géneros Esenbeckia y Raputia (Rutaceae), http://scielo.sld.cu/scielo.php?pid=S102847962013000400015\&script=sci_arttext; ISSN: 1028-4796, Rev. Cubana Plant. Med: 18(4), 638-653 (2013)

Cuca, L.E., C. Coy., E. Coy. y J. Lozano, Antibacterial activity of terpenes and alkaloids isolated from three Colombian plants, http://scielo.sld.cu/scielo.php?script=sci_arttext\&pid=S0034-75152011000200012; ISSN: 1561-2988, Rev. Cubana Farm: 45(2), 275-282 (2011)

Delmas, F. y otros seis autores, Synthesis and antileishmanial activity of (1,3-benzothiazol-2-yl) amino-9(10H)-acridinone derivatives, Eur J Med Chem: 39(8), 685-690 (2004)

Finney, J.D., Statistical logic in the monitoring of reactions to therapeutic drugs, Methods Inf Med: 10(4), 237$245(1971)$

Galeano, E., O. Thomas., D.L. Muñoz., S.M. Robledo., A. Martinez, Antiparasitic Bromotyrosine Derivatives from the Marine, Sponge Verongula rigida, doi: 10.3390/md9101902, Marine Drugs: 9(10), 1902-1913 (2011)

Giménez, A., A. y otros quince autores, Estudios químicos, biológicos y farmacológicos de Galipea longiflora KRAUSE, http://www.redalyc.org/articulo.oa?id=426339667015; ISSN: 0250-5460, Rev. Bol. Quim: 22(1), 94-107 (2005)

Guzman, M., G. Santafé., M. Salcedo., A. Angulo y O. Torres, Estudio químico y actividades antioxidante y bactericida de Ganoderma applanatum. Biotecnología en el Sector Agropecuario y Agroindustrial: 11(1), 8894 (2013)

Instituto Nacional de Salud, Boletín Epidemiológico No 39 de la Leishmania en Colombia 2015, http://www.ins.gov.co/boletinepidemiologico/Boletn\%20Epidemiolgico/2015\%20Boletin\%20epidemiologico\% 20semana\%2039.pdf. Acceso: 13 de Octubre (2015)

Insuasty, B. y otros doce autores, An efficient synthesis of new caffeine-based chalcones, pyrazolines and pyrazolo[3,4-b][1,4]diazepines as potential antimalarial, antitrypanosomal and antileishmanial agents, doi.org/10.1016/j.ejmech.2015.02.040, Eur J Med Chem: 93(1), 401-413 (2015)

Januário, A. H. y otros seis autores, Alcaloides $\beta$-indolopiridoquinazolínicos de Esenbeckia grandiflora MART. (Rutaceae), Quim. Nova: 32 (8), 2034-2038 (2009)

Macías, V. E., E. D. Coy. y L. E. Cuca, Análisis fitoquímico preliminar y actividad antioxidante, antiinflamatoria y antiproliferativa del extracto etanólico de corteza de Zanthoxylum fagara (L.) Sarg. (Rutaceae), Rev. Cubana Plant Med: 16 (1), 43-53 (2011)

Michael, J., Quinoline, quinazoline and acridone alkaloids, doi: 10.1039/b612168n, Nat. Prod. Rep: 25(1), 166-187 (2008)

Montaño, M. y G. Santafé, Evaluation of antioxidant activity of marine sponges from the Colombian Caribbean, Actu. Bio: 33(95), 173-175 (2011) 
Ngoumfo, R. y otros diez autores, In vitro cytotoxic activity of isolated acridones alkaloids from Zanthoxylum leprieurii Guill. et Perr, doi:10.1016/j.bmc.2010.03.040, Bioorganic \& Medicinal Chemistry: 18(10), 36013605 (2010)

Ovalle, C.E., L. Porras., M. Rey., M. Ríos. Y Y.C. Camargo, [Geographic distribution of Leishmania species isolated from patients at the National Institute of Dermatology Federico Lleras Acosta E.S.E., 1995-2005], Biomedica: 26 Suppl 1:145-51 (2006)

Patiño, O., Monophyllidin, A new alkaloid L-proline derivative from Zanthoxylum Monophyllum, doi:10.1016/j.phytol.2010.10.002, Phytochemistry Letters: 4(1), 22-25 (2011)

Pulido, S.A. y otros seis autores, Improvement of the green fluorescent protein reporter system in Leishmania spp. for the in vitro and in vivo screening of antileishmanial drugs, doi:10.1016/j.actatropica.2011.11.015, Acta Tropica: 122(1), 36- 45 (2012)

Sanchez, E. L, G.G. Santafé, O.L. Torres, D.L. Muños y S.M. Robledo. Compuestos sintéticos del tipo de estirilquinolínas con actividad leishmanicida y citotóxica. Biomédica: 34(4), 605-611 (2014)

Segura, R., A.V. Carrera., A.M. Cubides., A.M. Gomez y L.C. Rubiano, Falla Terapéutica en Leishmaniasis Valle del Cauca, Revista Medicina y Salud Fusm: 1(1), 30-32 (2013)

Torres, O.L, Estudio fitoquímico y de actividad biológica (antimalárica y antimicrobiana) de los extractos etanólicos de hoja, corteza y madera de Esenbeckia litoralis (Rutaceae), Tesis de Magíster, Dpto. De Química, Universidad Nacional de Colombia, Medellin, Colombia (2001)

Weniger, B., S.M. Roble., G.J. Arango., E. Deharo., R. Aragon. y V. Muñoz. Antiprotozoal activities of Colombian plants, doi.org/10.1016/S0378-8741(01)00346-4, J Ethnopharmacol, 78(2-3):193-200 (2001)

World Health Organization (WHO). Control of the leishmaniases: report of a meeting of the WHO Expert Commitee on the Control of Leishmaniases. Technical report 949 (2010), http://apps.who.int/iris/bitstream/10665/44412/1/WHO_TRS_949_eng.pdf. Acceso: 8 de Octubre, pp 508 (2015) 